\title{
Correlating the WFC and the IBIS hard X-ray surveys
}

\section{F. Capitanio*}

IASF-Roma INAF. Via Fosso del Cavaliere, 10000133 Rome Italy

E-mail: fiamma.capitanio@iasf-roma.inaf.it

\section{A. J. Bird}

School of Physics and Astronomy, University of Southampton, Highfield, Southampton, SO17 IBJ, UK

\section{A. Bazzano}

IASF-Roma INAF. Via Fosso del Cavaliere, 10000133 Rome Italy

\section{P. Ubertini}

IASF-Roma INAF. Via Fosso del Cavaliere, 10000133 Rome Italy

\begin{abstract}
During the operational life of BeppoSax, the Wide Field Camera observations covered almost the full sky at different epochs. The energy coverage overlap, the wide field of view and the imaging capabilities share many commonalities with IBIS, the gamma-ray telescope on board INTEGRAL satellite. We mosaicked all the available single WFCs observation images and then we searched the map produced for persistent and transient sources as it has been done for the IBIS survey. This work represents the first unbiased source list compilation produced in this way from the overall WFC data set. Similarity and differences with the IBIS results will be described.
\end{abstract}

The Extreme sky: Sampling the Universe above $10 \mathrm{keV}$ - extremesky2009,

October 13-17, 2009

Otranto (Lecce) Italy

\footnotetext{
*Speaker.
} 
Table 1: BeppoSAX WFCs characteristics compared with the IBIS/ISGRI ones.

\begin{tabular}{ccc}
\hline \hline parameter & WFC value & ISGRI value \\
\hline \hline Energy range & $3-28 \mathrm{keV}$ & $17 \mathrm{keV}-1 \mathrm{MeV}$ \\
\hline Energy resolution & $20 \%$ at $6 \mathrm{keV}$ & $8 \%$ at $100 \mathrm{keV}$ \\
\hline Field of view & $40^{\circ} \times 40^{\circ}(\mathrm{FWZR})$ & $29^{\circ} \times 29^{\circ}$ zero response \\
& $20^{\circ} \times 20^{\circ}(\mathrm{FWHM})$ & $\left(8.9^{\circ} \times 8.9^{\circ}\right.$ FCFOV) \\
\hline Angular Resolving Power & 5 & $12^{\prime}$ \\
\hline Source location accuracy & $<1$ arcminute & 3 arcminute at $100 \mathrm{keV}(5 \sigma)$ \\
& & 50 arcseconds at $100 \mathrm{keV}(50 \sigma)$ \\
\hline Sensitivity in $10^{3} s(5$ sigma) $)$ & $\sim 10$ mCrab $(3-28 \mathrm{keV})$ & $\sim 30$ mCrab $(20-40 \mathrm{keV})$ \\
\hline
\end{tabular}

\section{Introduction}

The two Wide Field Cameras (WFCs) [Jager et al. 1997] on board the BeppoSAX satellite [Boella et al. 1997a], were mounted 180 degrees away from each other and perpendicular to the pointing direction of the Narrow Field Instruments (NFI) looking at two different sky zones during any NFI pointing. In this way, during the 6 years of BeppoSAX operational life, the WFCs secondary mode observations covered almost all the sky, with at least one pointing (typically $100 \mathrm{ksec}$ duration). Moreover, two times a year the WFCs observed, as primary instrument, the Galactic Bulge region. The time devoted to this program corresponds to $8 \%$ of the total observing time of the satellite. Twelve such campaigns were carried out amounting to a total net exposure up to 6 Msec.

IBIS (the gamma-ray telescope on board INTEGRAL satellite) and WFCs are both coded mask instruments, they have a wide field of view and an observational strategy that favours the Galactic Centre zone. They are characterised by a complementary energy range, partially overlapped, and a good capability to detect transient sources thanks to their large field of view. The different characteristics of the two instruments are summarised in Table 1. We analysed all the available BeppoSAX Wide Field Camera pointings providing a mosaic of the images of all the single pointing observations and searched the map for sources using the same softwares and techniques developed for the IBIS survey [Bird et al. 2007, Bird et al. 2009].

Each excess found in the WFCs maps was searched for in the IBIS maps in order to better distinguish between structures of the IBIS survey mosaics and sources.

A comparison between the IBIS source catalogue and the WFC one, especially in the overlapping energy range of the two instruments, can be very useful in order to better understand the nature of the sources detected in the IBIS survey.

\section{WFC Sky Maps Production}

We analysed all the selected OPs (Observation Periods) with the WFC Data Analysis System, extracting the images for two energy ranges: $3-17 \mathrm{keV}$ and $17-28 \mathrm{keV}$. The latter band was selected to allow a direct comparison between WFCs and IBIS. The WFC data are organised in short 
observational periods (OP) of at least $100 \mathrm{ks}$. We selected all the WFC OPs available using the new archive of the INAF/IASF Institute of Rome. Compared to the results reported in Capitanio et al. 2008, the IASF archive now contains all the data collected both from the ASI DATA CENTER (ASDC) and from the old IASF archive. Even if we could not rescue some of the corrupted OPs, the amount of the analysed data reaches about $95 \%$ of the total amount of existing OPs. The collected data covered all the sky although not uniformly, in fact there are zones with higher exposure such as the Galactic Center and the zone around the Polaris star (and the "anti" Polaris zone). This is due to the observing strategy and manoeuvre techniques of the BeppoSAX satellite. The averaged exposure of the entire map is of about $4 \times 10^{6} \mathrm{~s}$ even if there are some regions with an exposure of two orders of magnitude less and others where the exposure reaches $\sim 2 \times 10^{7}$ s. Nevertheless the lower exposure regions are quite small covering few zones of the sky with a radius at least about 5 degrees. Figure 1 shows the exposure map of the total WFC OPs mosaic.

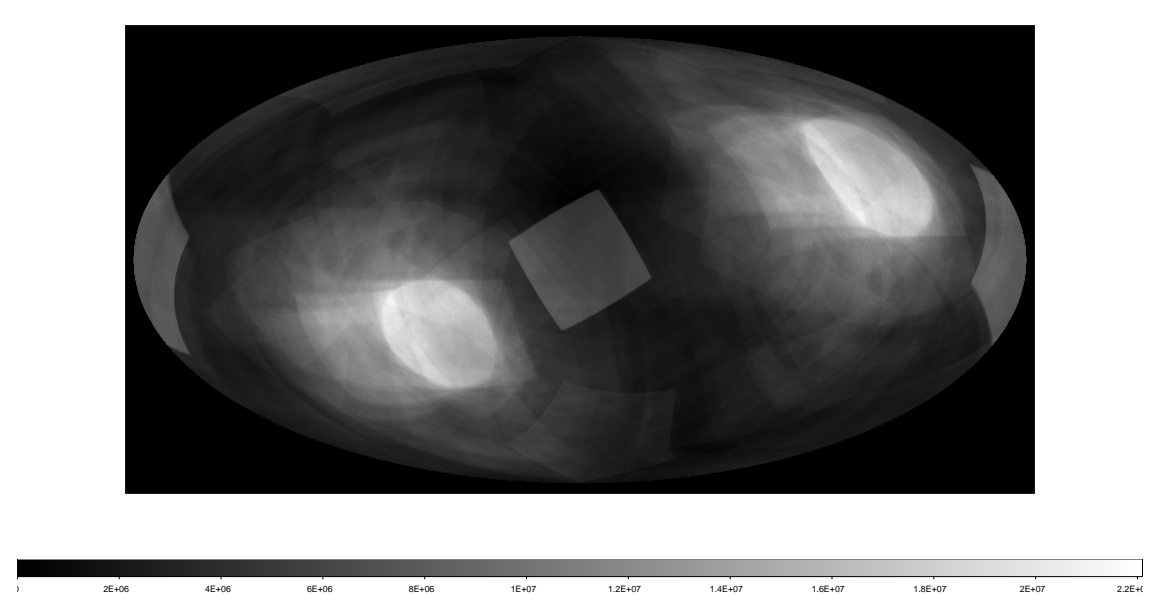

Figure 1: The WFC mosaic exposure map.

The WFC standard software uses the IROS method (Iterative Removal of Sources) to extract sources from the shadowgrams of the WFC coded masks. During the IROS procedures, we forced the software to extract a large number of sources for each OP image, effectively cleaning down into the noise. A mosaic of the images, for both energy ranges has been generated using the same software used for the IBIS survey [Bird et al. 2007, Bird et al. 2009]. In this way the possibly false detections extracted by "stressing" the WFC software have been eliminated. Two final maps have been obtained, Figure 2 shows the 3-17 keV WFC sky map. It is clear that the analysis of WFC data carried out so far has concentrated on locating transient sources [Verrecchia et al. 2007], whereas our methods allow a much more efficient detection of weak persistent sources.

\subsection{Source List Generation}

The source positions and fluxes were identified using a barycentering method to determine the centroid of the source profile. The mean flux of the sources was determined from the count rate at the position of the source maximum significance, using the same software developed for the IBIS survey. A second method based on SExtractor 2.4.4 software [Bertin and Arnouts 1996] has 


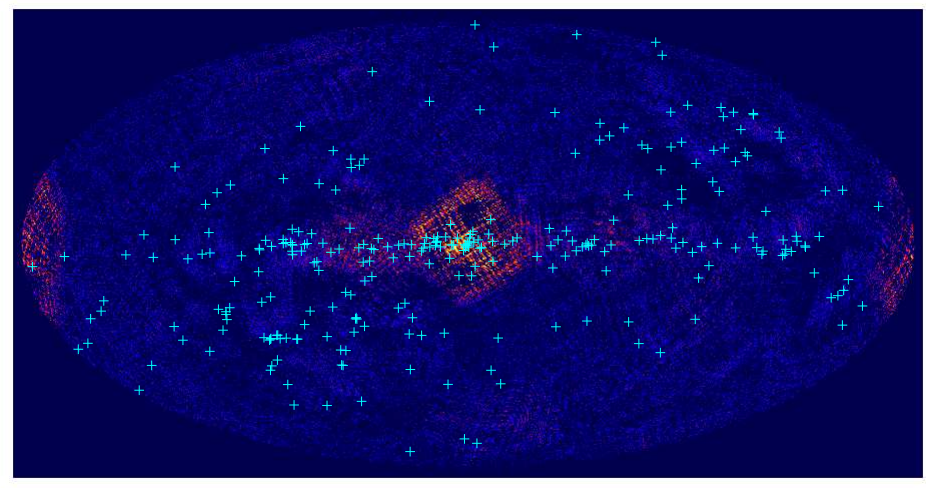

Figure 2: WFCs final mosaic between 3-17 keV. The crosses represent the detected sources positions

been used to cross check the results. The list of excesses was then checked manually. After all the checks the final list contains 336 excesses. Of these excesses 306 were identified and classified as sources while 30 do not have a firm identification yet. Of these 30 excesses, after a filtering and a visual inspection of the maps, only three have been selected as new source candidates. All the WFCs excesses were compared with the INTEGRAL survey 4 [Bird et al. 2009] lowest energy range maps, $17-30 \mathrm{keV}$ and $20-40 \mathrm{keV}$.

\section{Correlation between the IBIS and WFC Maps}

The INTEGRAL and WFC source lists have been compared, both for understanding systematic effects in the IBIS survey and to get more information on the characteristics of the sources, such as recurrency and duration of outbursts as well as spectral and time variability. The left panel of Figure 3 shows the different type distribution of the sources, with respect to their total number, for both the IBIS 4th catalogue [Bird et al. 2009] and this WFC catalogue. The distribution is different for the two instruments and this is mainly due to their different energy coverage, exposure and sensitivity: as the left panel of Figure 3 shows, the sources of the IBIS survey 4 catalogue present a higher percentage of unidentified sources with respect to the WFC catalogue. This is probably due to both the greater sensitivity and the less explored energy range of IBIS. Otherwise the WFCs catalogue lists a higher percentage of clusters of galaxies and low-mass X-ray binaries because of the softer energy spectra of the formers and the greater relative exposure of the WFC in the Galactic Centre for the latters.

In order to better understand the difference between the sources detected by IBIS and WFC, the right panel of Figure 3 shows the difference in $\%$ of the source types detected only by WFCs or by either IBIS and WFCs with respect to the total of WFCs sources. The diagram shows some remarkable differences: most of the LMXBs are present in the mosaics of both instruments. $10 \%$ of them, detected only by WFCs, were mostly off after the INTEGRAL launch as can be verified from the RXTE/ASM light curves. The same situation applies for the HMXBs: the ones detected only by WFCs were mostly off after the INTEGRAL launch. The number of transient sources in 

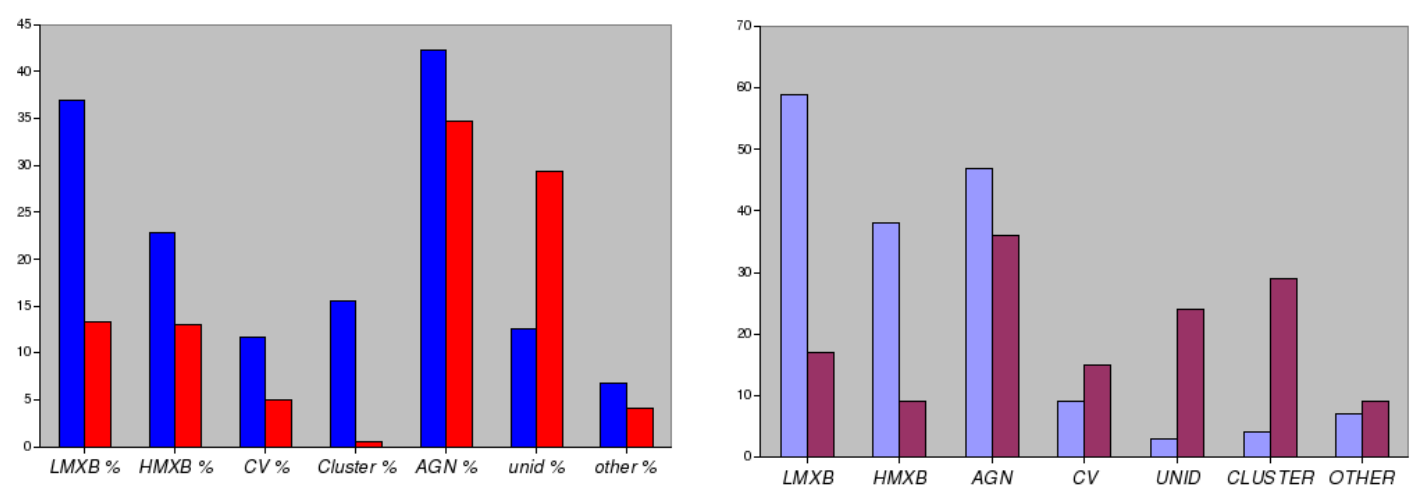

Figure 3: Left panel: Percentage of different source types detected in WFC (blue columns) and IBIS/ISGRI (red columns) catalogues. The question mark indicates the unidentified sources.Right panel: Percentage of different source types detected only by WFCs (pale blue columns) and by both WFCs and IBIS (claret columns). The question mark indicates the unidentified sources.

HMXB is indeed less than the one in LMXB, this is obviously reflected in the histogram of The right panel of Figure 3. The typical soft spectra of clusters of galaxies is probably the reason why this class of sources is mostly detected only by WFC.

Concerning AGN detected in the WFCs mosaics, about $30 \%$ of them has not been observed by IBIS, and this is principally due to different exposure time distribution of the two instruments.

\section{Comparison between our WFC catalogue and the previous one.}

On July 2007 a WFC catalogue was published [Verrecchia et al. 2007]. This work is based on the analysis of each single pointing observations and points mainly to the transient sources detection (a similar work, restricted to the galactic plane zone, was published in 2004 by Capitanio et al.). Our work is instead based on the searching of mosaic maps and is intended to identify persistent sources. For this reason our list of sources is slightly different from the one published in Verrecchia et al. 2007. The Verrecchia at al. catalogue contains 253 sources while our catalogue contains 306; 207 sources are present in both catalogues. The 46 sources reported only in Verrecchia et al. are all transient sources, while, as we expected, the 99 found only by us are mostly weak and persistent. It is important to notice that within these 99 sources there are 15 IGR sources ${ }^{1}$ and 10 SWIFT sources that have been discovered only after the end of the Beppo SAX mission.

A typical example of the different approach of our catalogue can be seen in Figure 4 that represents a zoom of the WFC 3-17 keV map on the Galactic Centre region. The white labelled sources shown in Figure 4 image are the sources reported in Verrecchia et al. 2007 catalogue, while the red ones are the sources reported in our catalogue. Most of the sources are detected by both catalogues, but for example, 1E1743.1-2843 is detected only by our catalogue. In fact 1E1743.12843 is a typical faint persistent source for which our work is optimised [Del Santo et al. 2006].

\footnotetext{
${ }^{1}$ i.e. sources detected for the first time by INTEGRAL
} 


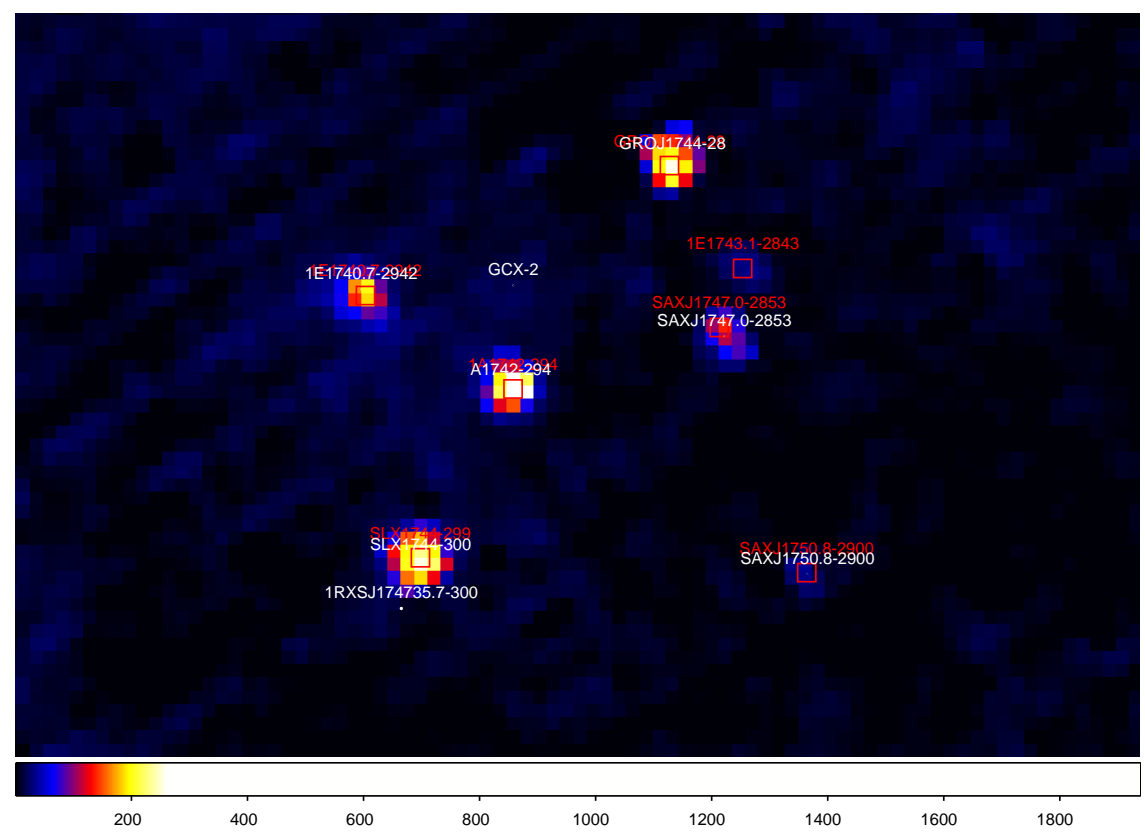

Figure 4: Zoom of the Galactic centre region 3-17 keV map. The sources found in the Verrecchia et al. catalogue are labelled in white colour while the sources found in our catalogue are labelled in red

\section{Conclusions}

This work has been made with the complete SAX/WFC dataset. We are still working on the final list of sources trying to refine the softwares used for the maps searching. In fact preliminary results indicate that detections at lower sigma level than in the previous analysis[Capitanio et al. 2008], can be considered as real sources We are also finalising the production of light curves for the brightest sources all along the six years of BeppoSAX operational life. The techniques developed for the IBIS survey have been successfully applied in this work to the WFC data, the same method could also be used with other coded mask instruments like INTEGRAL/JEM-X or SWIFT/BAT.

\section{References}

[Bertin and Arnouts 1996] Bertin, E., \& Arnouts, S., 1996, 117, 393.

[Boella et al. 1997a] Boella, G., Butler R.C., Perola, C. et al., 1997, A\&AS, 122, 299.

[Bird et al. 2009] Bird, A. J., Bazzano, A., Bassani, L., Capitanio, F. et al., 2009, ApJS in press

[Bird et al. 2007] Bird, A. J., Malizia, A., Bazzano, A. et al. 2007, ApJS, 170, 175.

[Capitanio et al. 2008] Capitanio, F., Bird, A. J., Ferici, M. et al. 2008, AIPC,1010, 257

[Capitanio et al. 2004] Capitanio, F., Bazzano, A., Cocchi, M., 2004, Nuc. Phys. B. 132, 580

[Del Santo et al. 2006] Del Santo, M., Sidoli, L., Bazzano, A., 2006, A\&A, 456, 1105.

[Verrecchia et al. 2007] Verrecchia, F., in't Zand, J. J. M., Giommi, P., A\&A 4727052007

[Jager et al. 1997] Jager, R., Mels, W.A., Brinkman, A.C. et al., 1997, A\&AS, 125, 557. 